\title{
DNA methylation signatures associated with cardiometabolic risk factors in children from India and The Gambia: results from the EMPHASIS study
}

\author{
Elie Antoun ${ }^{1 \dagger}$, Prachand Issarapu ${ }^{2 \dagger}$, Chiara di Gravio ${ }^{3}$, Smeeta Shrestha ${ }^{2,4}$, Modupeh Betts ${ }^{5}$, Ayden Saffari ${ }^{6}$, \\ Sirazul A. Sahariah ${ }^{7}$, Alagu Sankareswaran ${ }^{2}$, Manisha Arumalla ${ }^{2}$, Andrew M. Prentice ${ }^{5}$, Caroline H. D. Fall ${ }^{3 \dagger}$, \\ Matt J. Silver ${ }^{6 \dagger}$, Giriraj R. Chandak ${ }^{2+}$, Karen A. Lillycrop ${ }^{1,8^{*+}} \odot$ and the EMPHASIS study group
}

\begin{abstract}
Background: The prevalence of cardiometabolic disease (CMD) is rising globally, with environmentally induced epigenetic changes suggested to play a role. Few studies have investigated epigenetic associations with CMD risk factors in children from low- and middle-income countries. We sought to identify associations between DNA methylation (DNAm) and CMD risk factors in children from India and The Gambia.

Results: Using the Illumina Infinium HumanMethylation 850 K Beadchip array, we interrogated DNAm in 293 Gambian (7-9 years) and 698 Indian (5-7 years) children. We identified differentially methylated CpGs (dmCpGs) associated with systolic blood pressure, fasting insulin, triglycerides and LDL-Cholesterol in the Gambian children; and with insulin sensitivity, insulinogenic index and HDL-Cholesterol in the Indian children. There was no overlap of the dmCpGs between the cohorts. Meta-analysis identified dmCpGs associated with insulin secretion and pulse pressure that were different from cohort-specific dmCpGs. Several differentially methylated regions were associated with diastolic blood pressure, insulin sensitivity and fasting glucose, but these did not overlap with the dmCpGs. We identified significant cis-methQTLs at three LDL-Cholesterol-associated dmCpGs in Gambians; however, methylation did not mediate genotype effects on the CMD outcomes.
\end{abstract}

Conclusion: This study identified cardiometabolic biomarkers associated with differential DNAm in Indian and Gambian children. Most associations were cohort specific, potentially reflecting environmental and ethnic differences.

Keywords: EMPHASIS, DNA methylation, Epigenetics, Cardiovascular disease risk, Cardiometabolic risk factors, Early childhood risk factors

*Correspondence. kal@soton.acuk

†Elie Antoun and Prachand Issarapu: Joint first authors

${ }^{\dagger}$ Caroline H. D. Fall, Matt J. Silver, Giriraj R. Chandak, Karen A. Lillycrop: Joint senior authors

${ }^{1}$ School of Medicine, University of Southampton, Southampton, UK

Full list of author information is available at the end of the article

\section{Background}

Cardiometabolic disease (CMD) describes a range of conditions characterised by insulin resistance (IR), impaired glucose tolerance, dyslipidaemia and hypertension, risk factors for type 2 diabetes and cardiovascular disease (CVD). The increasing prevalence of CMDs poses a serious health burden. Although CMD is traditionally associated with high-income countries (HICs), original author(s) and the source, provide a link to the Creative Commons licence, and indicate if changes were made. The images or other third party material in this article are included in the article's Creative Commons licence, unless indicated otherwise in a credit line to the material. If material is not included in the article's Creative Commons licence and your intended use is not permitted by statutory regulation or exceeds the permitted use, you will need to obtain permission directly from the copyright holder. To view a copy of this licence, visit http://creativecommons.org/licenses/by/4.0/. The Creative Commons Public Domain Dedication waiver (http://creativeco mmons.org/publicdomain/zero/1.0/) applies to the data made available in this article, unless otherwise stated in a credit line to the data. 
prevalence has rapidly increased in low- and middleincome countries (LMICs) [1, 2]. Globally, the prevalence of type 2 diabetes has increased between 1980 and 2014 [3], but at higher rates in LMICs [1]. The prevalence of childhood hypertension in Central India is reported to be $6.8-7.0 \%$ [4] rising to $9.5 \%$ in Chennai [5], compared to $4 \%$ globally. Furthermore, mortality occurs earlier in LMICs, with the number of years spent living with these conditions increasing [2,3], escalating the societal and individual health burden. The rapid rise in CMD cannot be explained solely by fixed genetic factors, but suggests that environmental factors may contribute, including a change from traditional to western diets, increased intake of processed foods, urbanisation and reduced physical activity [6, 7]. Moreover, there is substantial evidence that early life environmental exposures during critical developmental windows modulate CMD risk [8, 9]. Exposure to persistent undernutrition, poor quality diets and a high burden of infectious diseases in utero and in early childhood are suggested to induce metabolic adaptations to aid survival. However, these adaptations may be detrimental in later life, limiting metabolic capacity in response to an obesogenic environment [10]. The early onset of cardiovascular and metabolic conditions in adults from LMICs compared to HICs may reflect such adverse early life adaptations.

The environment can influence phenotype through epigenetic processes. The most widely studied epigenetic mechanism is DNA methylation (DNAm), with evidence from both human and animal studies linking environmental exposures to DNAm and metabolic changes and altered CMD risk susceptibility [11-13]. In humans, candidate gene and epigenome-wide association studies (EWAS) have identified robust associations between DNAm and CMD traits in adulthood, which have been replicated across cohorts [14-16]. However, EWAS have primarily been carried out in HIC cohorts, with limited analysis of individuals from LMICs. As DNAm is influenced by both the environment and genotype [17], the extent to which methylation markers of CMD traits from HIC can be extrapolated to LMICs is unknown. Moreover, previous EWAS have focussed on CMD-associated DNAm changes in adults. Limited studies have examined DNAm in children, where the influence of early life environmental exposures may be stronger, with the possibility to detect methylation signatures associated with sub-clinical changes in metabolic function before disease onset.

In this study, we analysed DNAm in children from the EMPHASIS study [18] (Epigenetic Mechanisms linking Pre-conceptional nutrition and Health ASsessed in India and sub-Saharan Africa; ISRCTN14266771) which includes two LMIC cohorts, one each from India and The Gambia.
Previously, we investigated the effect of maternal micronutrient supplementation on DNAm in their children [19]. Here, we sought to investigate associations of DNAm with cardiometabolic risk markers in children from each cohort, and in both cohorts combined through a meta-analysis. We also examined the potential influence of genetic variants and maternal micronutrient intervention at associated loci.

\section{Results \\ Cohort characteristics}

The characteristics of the children in the two cohorts are summarised in Table 1 and stratified by sex in Additional file 1: Table S1. There were 289 Gambian children $(53.6 \%$ male), with a median age of 9.0 years and 686 Indian children $(55.1 \%$ male), with a median age of 5.8 years. Mean blood pressure (systolic, diastolic and pulse pressure) was generally lower in the Indian children compared to the Gambian children. The Indian children also showed lower fasting, 30-min and 120-min glucose levels during an OGTT, whereas the Gambian children showed lower fasting insulin levels. Triglyceride and LDL levels were higher in the Indian children, whereas HDL levels were higher in the Gambian children.

\section{Gambian EWAS}

DNA methylation was examined using the Illumina Infinium HumanMethylation $850 \mathrm{~K}$ Beadchip array in peripheral blood samples from the Gambian children, and robust linear regression used to identify associations between DNA methylation and concurrent cardiometabolic risk factors. A full list of significant $\mathrm{dmCpGs}$ $(\mathrm{FDR}<0.05)$ can be found in Table 2, alongside equivalent statistics from the Indian cohort. There were no significant sex interactions with the dmCpGs identified in the Gambian children. Further details are described below.

\section{Blood pressure}

There were three significant DNAm associations with systolic blood pressure (SBP) (Fig. 1a). The two most significant dmCpGs were cg13455829 in the body of the Mediator Complex Subunit 22 (MED22) gene $(\mathrm{FDR}=0.015$, Fig. 1b); and cg22671726 within the Egl-9 Family Hypoxia Inducible Factor 2 (EGLN2) gene $(\mathrm{FDR}=0.015$, Fig. 1c). A $1 \mathrm{mmHg}$ SBP increase was associated with a $0.054 \%$ increase in DNAm at cg13455829 $(95 \% \mathrm{CI}=0.03,0.07)$ and a $0.017 \%$ decrease in methylation of $\operatorname{cg} 22671726(95 \% \mathrm{CI}=-0.02,-0.01)$. These $\mathrm{dmCpGs}$ were not associated with SBP in the Indian children. There were no significant associations with diastolic blood pressure (DBP) or pulse pressure (PP). 
Table 1 Cohort characteristics

\begin{tabular}{|c|c|c|c|c|c|}
\hline & $\begin{array}{l}\text { The Gambia } \\
N\end{array}$ & & $\begin{array}{l}\text { India } \\
N\end{array}$ & & $P$ value \\
\hline$\%$ Male $(N)$ & 289 & $53.6 \%(155)$ & 686 & $55.1 \%(378)$ & $p=0.879$ \\
\hline$\%$ Maternal intervention $(N)$ & 289 & $46.3 \%(140)$ & 686 & $46.8 \%(321)$ & $p=0.839$ \\
\hline Age (years) & 289 & $9.0(8.6-9.2)$ & 686 & $5.8(5.6-6.0)$ & $* * * * p<2.2 \times 10^{-16}$ \\
\hline WHO weight-for-age Z-score & 289 & $-1.37 \pm 0.91$ & 686 & $-1.70 \pm 1.07$ & ${ }^{* *} p=6.36 \times 10^{-4}$ \\
\hline$\%$ Underweight $(N)$ & 289 & $20.8 \%(60)$ & 686 & $40.2 \%(276)$ & ${ }^{* * *} p=2.78 \times 10^{-8}$ \\
\hline WHO height-for-age Z-score & 289 & $-0.72 \pm 0.85$ & 686 & $-1.01 \pm 0.96$ & ${ }^{* * *} p=2.13 \times 10^{-9}$ \\
\hline $\mathrm{BMI}\left(\mathrm{Kg} / \mathrm{m}^{2}\right)$ & 289 & $14.4 \pm 1.3$ & 686 & $13.4 \pm 1.4$ & $* * * * p<2.2 \times 10^{-16}$ \\
\hline$\%$ Stunted $(N)$ & 289 & $7.6 \%(22)$ & 686 & $15.3 \%(105)$ & ${ }^{* *} p=0.002$ \\
\hline$\%$ Wasted $(N)$ & 289 & $22.5 \%(65)$ & 686 & $37.2 \%(255)$ & ${ }^{* * *} p=2.19 \times 10^{-5}$ \\
\hline \multicolumn{6}{|l|}{ Blood pressure } \\
\hline Systolic blood pressure $(\mathrm{mmHg})$ & 287 & $110.0 \pm 8.2$ & 682 & $92.1 \pm 8.5$ & $p=0.464$ \\
\hline Diastolic blood pressure (mmHg) & 289 & $64.4 \pm 8.1$ & 682 & $56.0 \pm 7.5$ & $p=0.978$ \\
\hline Pulse pressure (mmHg) & 287 & $45.6 \pm 7.5$ & 682 & $36.1 \pm 6.0$ & $p=0.598$ \\
\hline \multicolumn{6}{|l|}{ OGTT } \\
\hline Fasting glucose (mmol/l) & 280 & $4.81 \pm 0.56$ & 669 & $4.71 \pm 0.50$ & $p=0.857$ \\
\hline 30-min glucose $(\mathrm{mmol} / \mathrm{l})$ & 276 & $7.09 \pm 1.40$ & 663 & $6.84 \pm 1.58$ & $p=0.769$ \\
\hline 120-min glucose $(\mathrm{mmol} / \mathrm{l})$ & 281 & $5.27 \pm 0.95$ & 651 & $4.69 \pm 0.90$ & $p=0.823$ \\
\hline Fasting insulin (pmol/l) & 287 & $19.2 \pm 11.6$ & 674 & $30.2 \pm 39.9$ & $p=0.908$ \\
\hline 30-min insulin (pmol/l) & 271 & $187.0 \pm 116$ & 656 & $170.1 \pm 132.1$ & $p=0.146$ \\
\hline HOMA2-S & 278 & $0.44 \pm 0.15$ & 665 & $0.64 \pm 0.66$ & $p=0.332$ \\
\hline Insulinogenic index & 257 & $8.64 \pm 37.0$ & 643 & $9.99 \pm 51.6$ & $p=0.360$ \\
\hline Insulinogenic index adjusted for HOMA2-S & 257 & $21.5 \pm 98.3$ & 643 & $23.5 \pm 130.0$ & $p=0.489$ \\
\hline \multicolumn{6}{|l|}{ Blood lipids } \\
\hline Triglycerides (mmol/l) & 270 & $0.62 \pm 0.22$ & 674 & $0.89 \pm 0.34$ & $p=0.896$ \\
\hline $\mathrm{HDL}-\mathrm{C}(\mathrm{mmol} / \mathrm{l})$ & 270 & $1.18 \pm 0.29$ & 674 & $1.03 \pm 0.22$ & $p=0.256$ \\
\hline LDL-C (mmol/l) & 261 & $2.18 \pm 0.59$ & 673 & $2.31 \pm 0.63$ & $p=0.668$ \\
\hline
\end{tabular}

Figures are either median (IQR) or mean \pm sd unless otherwise specified. $P$ values are from Mann-Whitney $U$ test for continuous outcomes and Chi-squared test of independence for categorical outcomes. Stunted defined as height $<-2$ SD below WHO height-for-age reference mean. Wasted defined as $<-2$ SD below WHO reference BMI-for-age mean. WHO =World Health Organisation; BMI=Body Mass Index; OGTT=Oral Glucose Tolerance Test; HOMA2-S =Insulin Sensitivity; HDL-C = High-Density Lipoprotein Cholesterol; LDL-C = Low-Density Lipoprotein Cholesterol

\section{Glucose levels}

There were no significant associations of DNAm with children's fasting, 30-min or 120-min glucose levels.

\section{Insulin levels}

There were two dmCpGs associated with fasting insulin levels: cg22388948 in the body of the Family with Sequence Similarity 46 Member A (FAM46A) gene $(\mathrm{FDR}=0.014)$; and cg13934266 in the body of the LysM Domain Containing $2($ LYSMD2) gene $(F D R=0.022)$. A $1 \mathrm{pmol} / \mathrm{l}$ increase in fasting insulin was associated with a $1.37 \%$ increase in methylation of cg22388948 (95\% $\mathrm{CI}=0.81,1.94)$ and with a $0.13 \%$ decrease in methylation of $\operatorname{cg} 13934266(95 \% \mathrm{CI}=-0.18,-0.09)$. These $\mathrm{dmCpGs}$ were not associated with fasting insulin levels in the Indian children. There were no significant associations with insulin sensitivity, or insulinogenic index, unadjusted or adjusted for HOMA2-S, in the Gambian children.

\section{Lipid levels}

DNAm at CpG cg15237100 located in an intergenic region on chromosome 15 was associated with triglyceride levels $(F D R=0.031)$. A $0.1 \mathrm{mmol} / \mathrm{l}$ increase in triglycerides was associated with a $4.4 \%$ decrease in methylation of $\operatorname{cg} 15237100(95 \% \mathrm{CI}=-0.63,-0.25)$. There were six dmCpGs associated with LDL-Cholesterol levels. The two most significant dmCpGs were cg01469688 in the promoter of the Suppressor Of Cancer Cell Invasion $(S C A I)$ gene $(\mathrm{FDR}=0.004)$, and $\operatorname{cg} 06952751$ in the promoter of the C18orf8 gene $(\mathrm{FDR}=0.004)$, with a $0.1 \mathrm{mmol} / \mathrm{l}$ increase in LDL-Cholesterol associated with a $1.3 \%$ and $3.8 \%$ increase in methylation of $\operatorname{cg} 01469688$ and 
Table 2 List of significant differentially methylated CpGs associated with various CMD markers

\begin{tabular}{|c|c|c|c|c|c|c|c|c|c|}
\hline \multirow[t]{2}{*}{ Traits } & \multirow[t]{2}{*}{ CpG ID } & \multirow[t]{2}{*}{ Genomic location (hg19) } & \multicolumn{3}{|c|}{ Gambian } & \multicolumn{3}{|l|}{ Indian } & \multirow[t]{2}{*}{ Gene } \\
\hline & & & Coef & $P$ value & FDR & Coef & $P$ value & $\overline{\text { FDR }}$ & \\
\hline \multicolumn{10}{|l|}{ Gambian dmCpGs } \\
\hline \multirow[t]{3}{*}{ Systolic blood pressure } & $\operatorname{cg} 13455829$ & chr9:136213445 & 0.054 & $3.04 \times 10^{-08}$ & 0.015 & -0.002 & 0.747 & 0.997 & MED22 \\
\hline & cg22671726 & chr19:41305423 & -0.017 & $3.74 \times 10^{-08}$ & 0.015 & 0.002 & 0.515 & 0.991 & EGLN2 \\
\hline & cg00368636 & chr7:64734674 & 0.040 & $1.64 \times 10^{-07}$ & 0.044 & 0.005 & 0.39 & 0.989 & Intergenic \\
\hline \multirow[t]{2}{*}{ Fasting insulin } & cg22388948 & chr6:82460558 & 1.373 & $1.78 \times 10^{-08}$ & 0.014 & 0.077 & 0.598 & 0.993 & FAM46A \\
\hline & cg13934266 & chr15:52029533 & -0.132 & $5.48 \times 10^{-08}$ & 0.022 & 0.014 & 0.279 & 0.979 & LYSMD2 \\
\hline Triglycerides & cg15237100 & chr15:69755217 & -0.439 & $5.32 \times 10^{-08}$ & 0.031 & -0.024 & 0.318 & 0.955 & $\begin{array}{l}\text { RP11-279F6.1/ } \\
\text { RP11-253M7.4 }\end{array}$ \\
\hline \multirow[t]{6}{*}{ LDL-C } & cg01469688 & chr9:127905934 & 0.132 & $8.74 \times 10^{-09}$ & 0.004 & 0.041 & 0.019 & 0.916 & SCAl \\
\hline & cg06952751 & chr18:21083211 & 0.384 & $9.33 \times 10^{-09}$ & 0.004 & 0.009 & 0.999 & 0.999 & C18orf8 \\
\hline & cg27229251 & chr7:2680532 & 0.494 & $2.29 \times 10^{-08}$ & 0.006 & 0.015 & 0.732 & 0.996 & TTYH3 \\
\hline & cg13135286* & chr1:218302163 & -4.858 & $5.07 \times 10^{-08}$ & 0.010 & 0.033 & 0.98 & 0.999 & Intergenic \\
\hline & cg13819288 & chr2:119898838 & -2.259 & $2.20 \times 10^{-07}$ & 0.035 & 0.163 & 0.392 & 0.987 & Intergenic \\
\hline & cg07988415 & chr4:153291285 & 2.748 & $3.12 \times 10^{-07}$ & 0.042 & 0.303 & 0.328 & 0.983 & FBXW7 \\
\hline \multicolumn{10}{|l|}{ Indian dmCpGs } \\
\hline HOMA2-S & cg10304969 & chr1:25795613 & 0.154 & 0.387 & 0.986 & -0.734 & $2.27 \times 10^{-08}$ & 0.018 & TMEM57 \\
\hline $\begin{array}{l}\text { Insulinogenic index } \\
\text { adjusted for HOMA2-S }\end{array}$ & cg22982428 & chr2:216402618 & -0.010 & 0.948 & 0.999 & 0.302 & $2.68 \times 10^{-08}$ & 0.022 & Intergenic \\
\hline $\mathrm{HDL}-\mathrm{C}$ & cg04988216 & chr1:64471626 & -0.223 & 0.190 & 0.974 & -0.956 & $2.36 \times 10^{-08}$ & 0.019 & $R O R 1$ \\
\hline
\end{tabular}

The gene name corresponds to the nearest gene as annotated in the Illumina EPIC array manifest. CMD = cardiometabolic disease; Coef $=$ regression beta coefficient; $\mathrm{FDR}=$ Benjamini-Hochberg false discovery rate; LDL-C = Low-Density Lipoprotein Cholesterol; HOMA2-S = Insulin Sensitivity; HDL-C = High-Density Lipoprotein cholesterol; $\mathrm{dmCpG}=$ differentially methylated $\mathrm{CpG}$. Regression coefficients were calculated using beta values in the regression models for the dmCpGs to obtain interpretable coefficients

*Denotes the association between the CpG and outcome was confounded by genotype

cg06952751, respectively (cg01469688: $95 \% \mathrm{CI}=0.09$, 0.18; cg06952751: $95 \% \mathrm{CI}=0.25,0.52)$. There were no associations between DNAm and HDL-Cholesterol levels in the Gambian children.

\section{Indian EWAS}

Table 2 lists all the significant dmCpGs from the Indian EWAS alongside equivalent statistics in the Gambian cohort. Some evidence of significant sex interactions was identified in the Indian children. Further details are described below.

\section{Blood pressure}

There were no significant associations between DNAm and SBP, DBP or PP in the Indian cohort.

\section{Glucose levels}

No significant associations were detected between DNAm and child's fasting, 30-min or 120-min glucose levels.

\section{Insulin levels}

There were no associations between DNAm and child's fasting insulin and insulin 30-min after an OGTT. The
CpG cg10304969, in the body of the Transmembrane protein 57 (TMEM57) gene, was associated with insulin sensitivity $(F D R=0.018)$, where a 1 unit decrease in HOMA2-S was associated with a $0.73 \%$ decrease in methylation (95\% CI $=-0.98,-0.049)$. cg22982428, in an intergenic region on chromosome 2, was associated with insulinogenic index adjusted for HOMA2-S $(F D R=0.022)$, where a 1 unit increase was associated with a $0.3 \%$ increase in methylation $(95 \% \mathrm{CI}=0.19,0.41)$. These CpGs were not significant in the Gambian cohort. There were no dmCpGs associated with the insulinogenic index in the Indian cohort.

\section{Lipid levels}

There were no significant associations with triglyceride or LDL-Cholesterol levels. One CpG was associated with HDL-Cholesterol levels (Fig. 2a); cg04988216 in the body of the Receptor Tyrosine Kinase Like Orphan Receptor 1 (ROR1) gene (FDR $=0.019$, Fig. 2b), where a $1 \mathrm{mmol} / \mathrm{l}$ increase in HDL-C was associated with a $0.96 \%$ decrease in methylation $(95 \% \mathrm{CI}=-1.27$, $-0.64)$. Figure $2 \mathrm{~b}$ suggests this association may be influenced by two outlier samples with a lower methylation beta value relative to the rest of the samples. The 


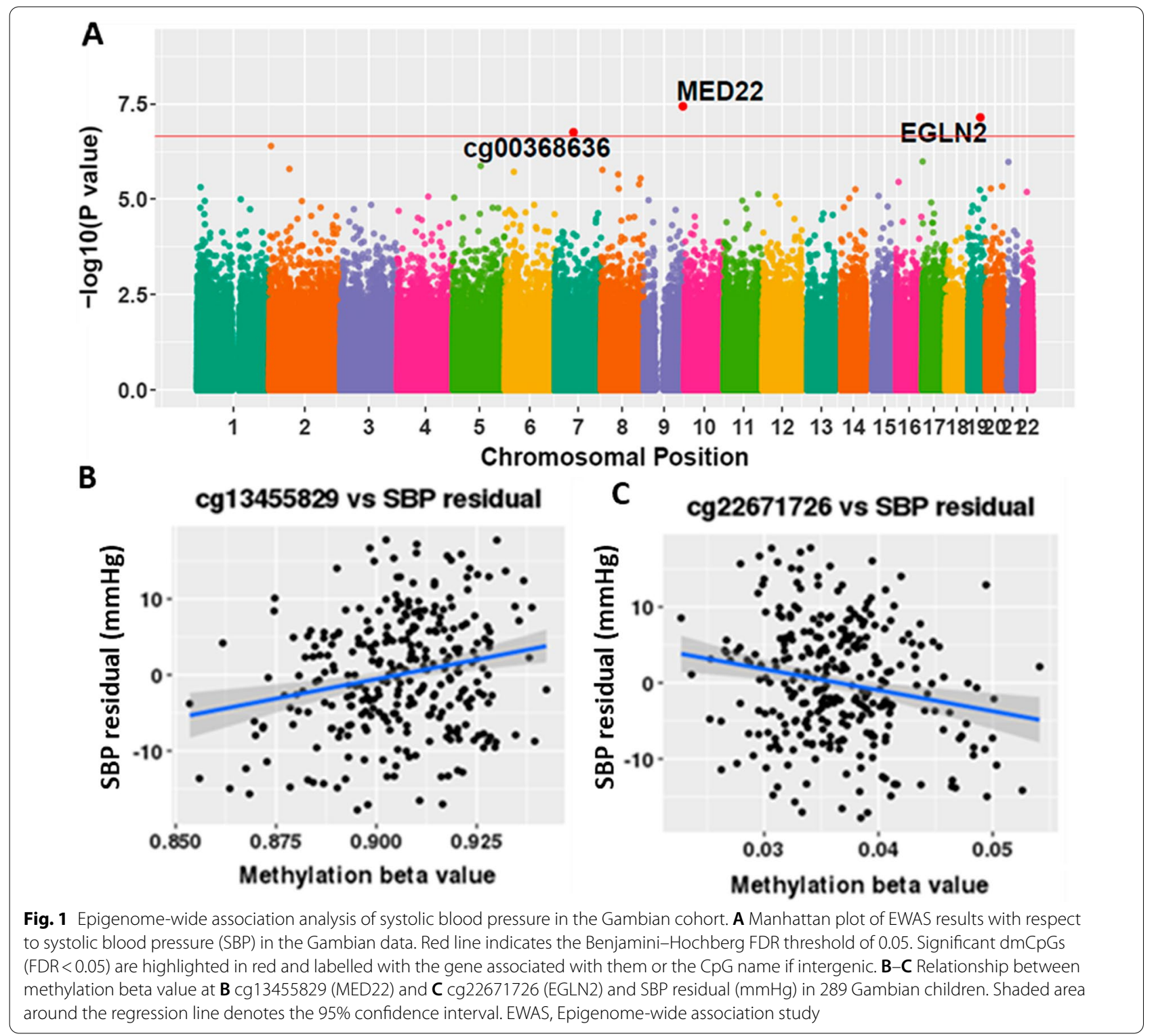

association was more significant (FDR $=0.003$, Fig. 2c) after removal of these two samples. Furthermore, methylation at cg04988216 in ROR1 gene showed a significant interaction with sex $\left(p=2.37 \times 10^{-3}\right.$, Fig. 2d), with methylation significantly associated with HDL-Cholesterol levels in the females $\left(p=2.67 \times 10^{-6}\right)$ but not the males $(p=0.24)$. The $\mathrm{CpG}$ cg04988216 was not significant in the Gambian children.

\section{Differentially methylated regions}

To identify regional differences in DNA methylation, DMRcate was used to identify DMRs associated with the

\section{(See figure on next page.)}

Fig. 2 Epigenome-wide association analysis of high-density lipoprotein cholesterol in the Indian cohort. A Manhattan plot of EWAS results with respect to high-density lipoprotein cholesterol (HDL-C) levels in the Indian data. Red line indicates the FDR threshold of 0.05 . Significant $\mathrm{dmCpGs}$ $($ FDR $<0.05)$ are highlighted in red and labelled with the gene associated with it. B Relationship between methylation beta value at cg04988216 (ROR1) and HDL-C residual (mmol/l) in 674 Indian children. Two outlier samples are highlighted in red. C Relationship between methylation beta value at cg04988216 (ROR1) and HDL-C residual (mmol/l) in 672 Indian children after removal of the two outlier samples highlighted in C. D Relationship between methylation at cg04988216 and HDL-C residuals ( $\mathrm{mmol} / \mathrm{l})$ in the male and female children separately. Shaded area around the regression lines denotes the 95\% confidence interval band. EWAS, Epigenome-wide association study 

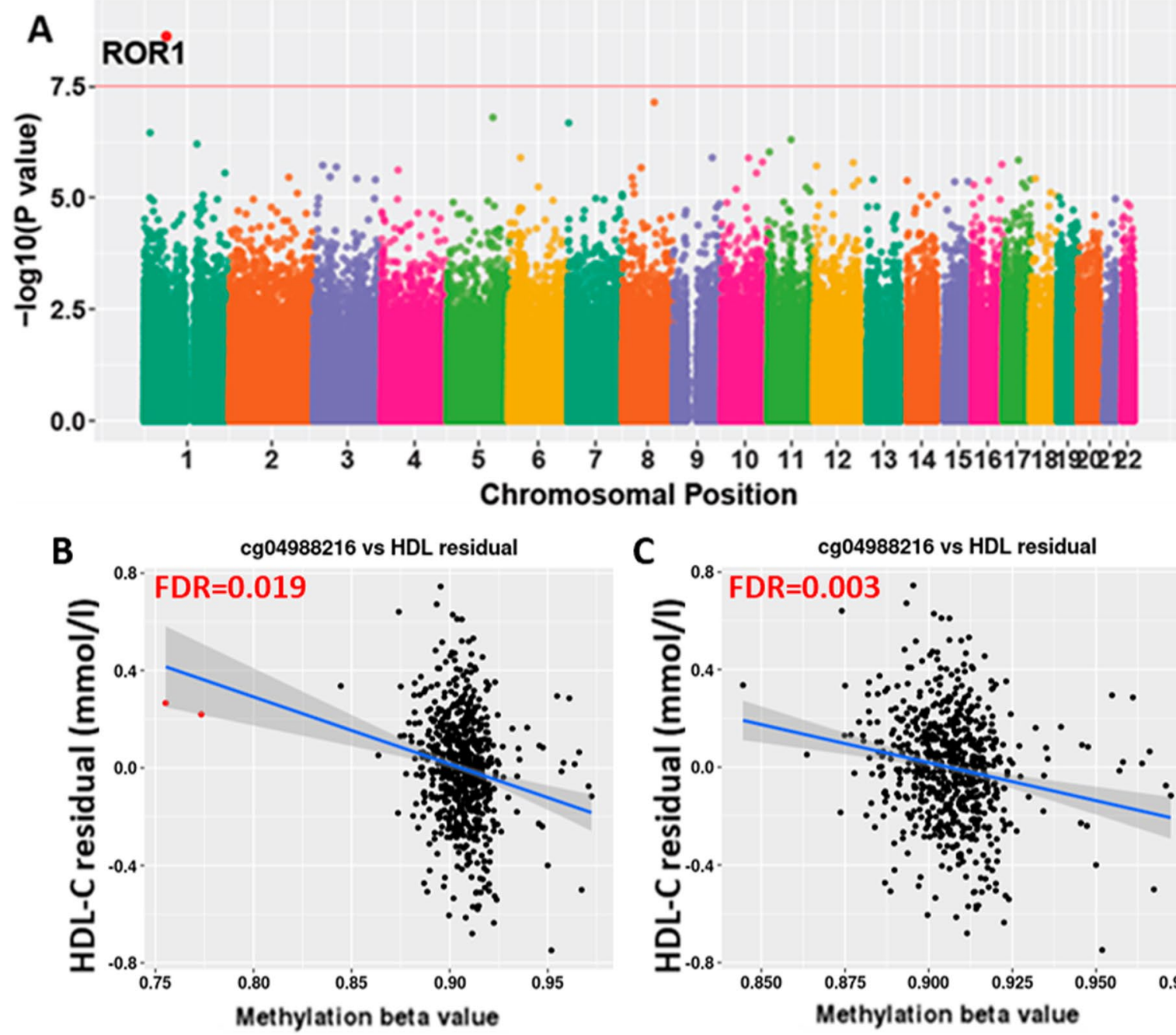

C cg04988216 vs HDL residual

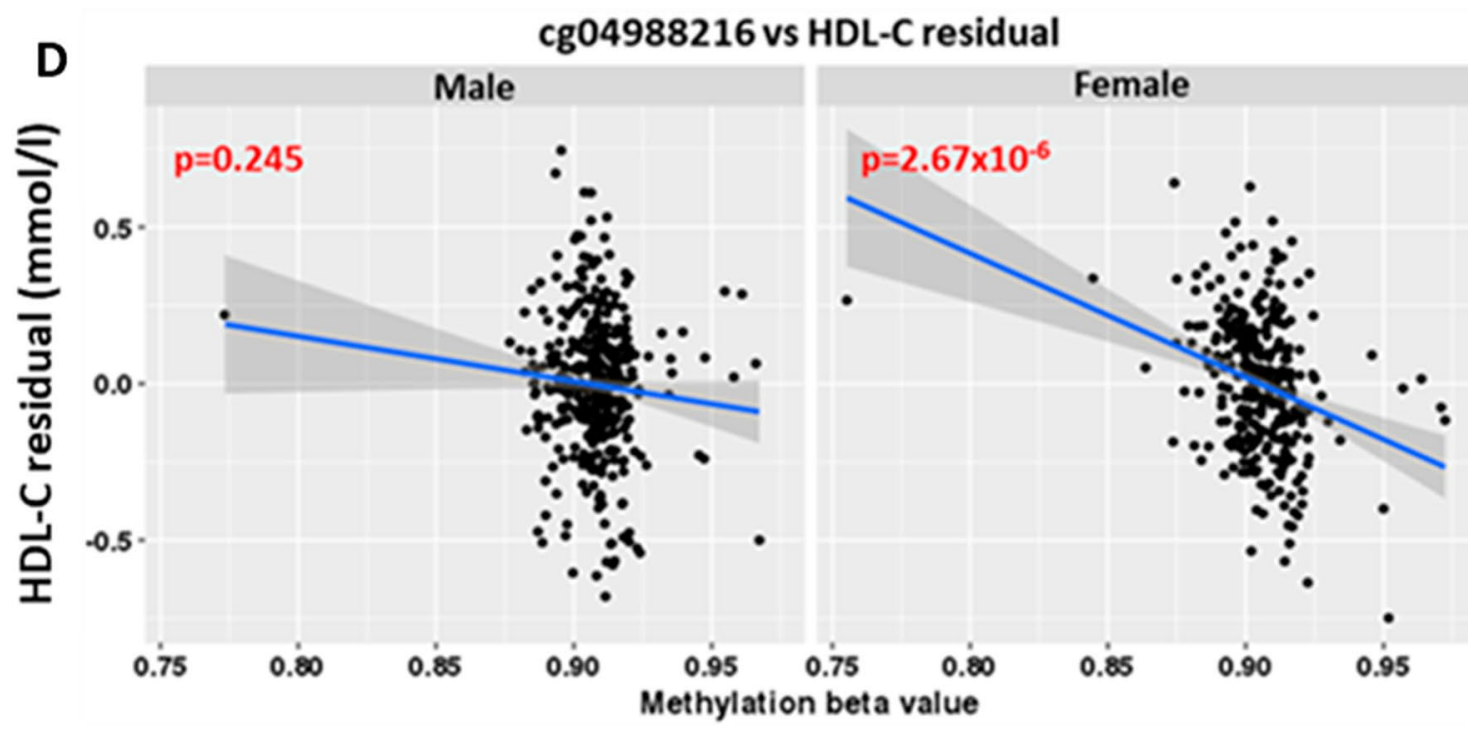

Fig. 2 (See legend on previous page.) 
CMD markers in the children (Table 3). In the Gambian children, there was one 69 bp DMR comprising three CpGs associated with DBP (Stouffer $<0.05$ ), located $1 \mathrm{~kb}$ upstream from the transcriptional start site of the Ephrin A1 (EFNA1) gene. One 576 bp DMR comprising nine CpGs was associated with fasting insulin levels, located in the promoter of the C8orf31 gene. In the Indian children, one 119 bp DMR consisting of two CpGs in an intergenic region on chromosome 20 was associated with fasting glucose. Eight DMRs were significantly associated with HOMA2-S, with the top DMR comprising two CpGs located in a $13 \mathrm{bp}$ intergenic region on chromosome 22. Of the DMRs identified, there were no overlaps between the cohorts. Furthermore, no DMRs were in close proximity to identified dmCpGs.

\section{Meta-analysis}

A meta-analysis was carried out to identify associations between DNA methylation and cardiometabolic outcomes common across both cohorts (Table 4). There were two dmCpGs significantly associated with the insulinogenic index (Fig. 3a, b). These were: cg04859490 $\left(\mathrm{FDR}=0.029, \mathrm{HetI}^{2}=0\right.$, Fig. 3c) in intron 8 of the Carboxypeptidase A4 (CPA4) gene; and cg00363845 $\left(\mathrm{FDR}=0.029\right.$, Het $I^{2}=51.7$, Fig. 3d) in intron 1 of the GTP binding protein 3 (GTPBP3) gene. Figure 3c suggests that cg04859490 may be influenced by genotype in the Indian cohort, and we did find evidence of methQTL effects in trans at this locus, although these were not genome-wide significant (Additional file 1: Table S2). In a sensitivity analysis, inclusion of genotype at these nominally associated SNPs into the regression models did not influence the effect size of the association between cg04859590 and the insulinogenic index in the Indian children (Additional file 1: Table S3). One dmCpG was found to be significantly associated with pulse pressure in the meta-analysis: $\operatorname{cg} 14997376\left(\right.$ Het $\left.I^{2}=0\right)$ in exon 39 of the Citron Rho-interacting kinase (CIT) gene.

\section{Links to early environment and preconception nutritional supplementation}

Various environmental exposures in early life have been linked to DNAm changes in children [13, 20, 21]. The mothers in both cohorts received a nutritional intervention pre- and during pregnancy [19]. Therefore, we examined whether the identified $\mathrm{dmCpGs}$ were associated with this intervention. None of the $\mathrm{dmCpGs}$ were associated with the intervention. Season of conception (SoC) is associated with DNA methylation signatures in Gambian children [22]. In the Gambian cohort, inclusion of SoC as a covariate did not influence the significance of the primary associations between CMD markers and DNAm at the $\mathrm{dmCpGs}$, and no significant interaction between $\mathrm{SoC}$ and DNAm was observed.

Table 3 List of significant differentially methylated regions associated with various CMD markers

\begin{tabular}{|c|c|c|c|c|c|c|}
\hline Chromosome & Start position & End position & Width (bp) & No. of CpGs & $\begin{array}{l}\text { Stouffer } \\
p \text { value }\end{array}$ & Associated gene \\
\hline \multicolumn{7}{|l|}{ Gambian } \\
\hline \multicolumn{7}{|c|}{ Systolic blood pressure } \\
\hline chr1 & $155,099,264$ & $155,099,332$ & 69 & 3 & 0.039 & EFNA1 \\
\hline \multicolumn{7}{|l|}{ Fasting insulin } \\
\hline chr8 & $144,120,106$ & $144,120,681$ & 576 & 9 & 0.010 & C8orf31 \\
\hline \multicolumn{7}{|l|}{ Indian } \\
\hline \multicolumn{7}{|l|}{ Fasting glucose } \\
\hline chr20 & $49,072,540$ & $49,072,658$ & 119 & 2 & 0.026 & Intergenic \\
\hline \multicolumn{7}{|l|}{ HOMA2-S } \\
\hline chr22 & $33,964,297$ & $33,964,309$ & 13 & 2 & 0.011 & Intergenic \\
\hline chr20 & $62,133,588$ & $62,133,626$ & 39 & 3 & 0.023 & RP4-697K14.3 \\
\hline chr3 & $194,866,144$ & $194,866,280$ & 137 & 2 & 0.024 & RN7SL36P \\
\hline chr1 & $79,088,559$ & $79,088,769$ & 211 & 2 & 0.030 & Intergenic \\
\hline chr6 & $150,071,069$ & $150,071,503$ & 435 & 3 & 0.037 & PCMT1 \\
\hline chr1 & $6,059,070$ & $6,059,204$ & 135 & 2 & 0.047 & Intergenic \\
\hline chr1 & $240,861,557$ & $240,861,571$ & 15 & 2 & 0.049 & Y_RNA.12 \\
\hline chr10 & $1,205,222$ & $1,205,942$ & 721 & 10 & 0.050 & LINC00200 \\
\hline
\end{tabular}

Genomic coordinates are all from the hy 19 build of the genome. $\mathrm{CMD}=$ cardiometabolic disease; $\mathrm{bp}=$ base pairs; HOMA2-S=Insulin Sensitivity 


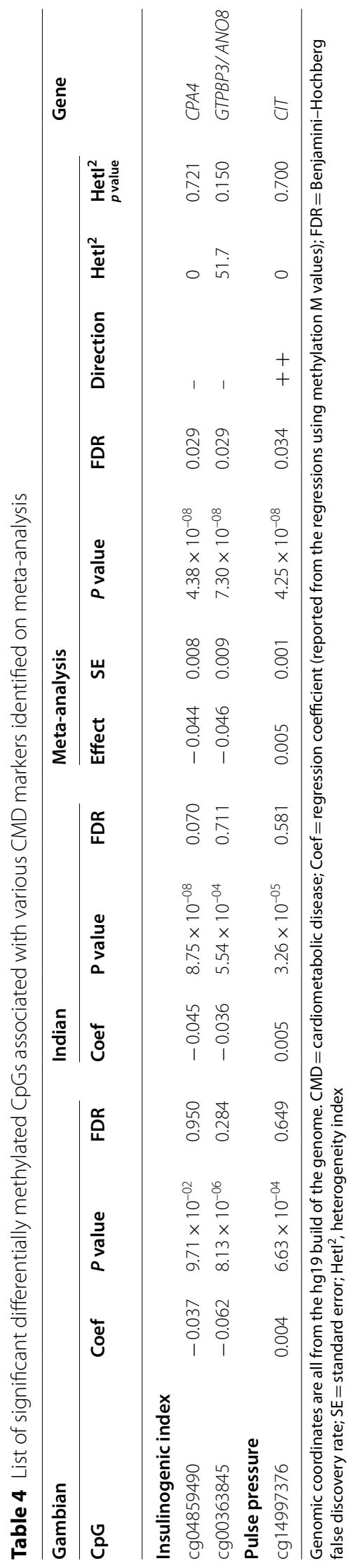




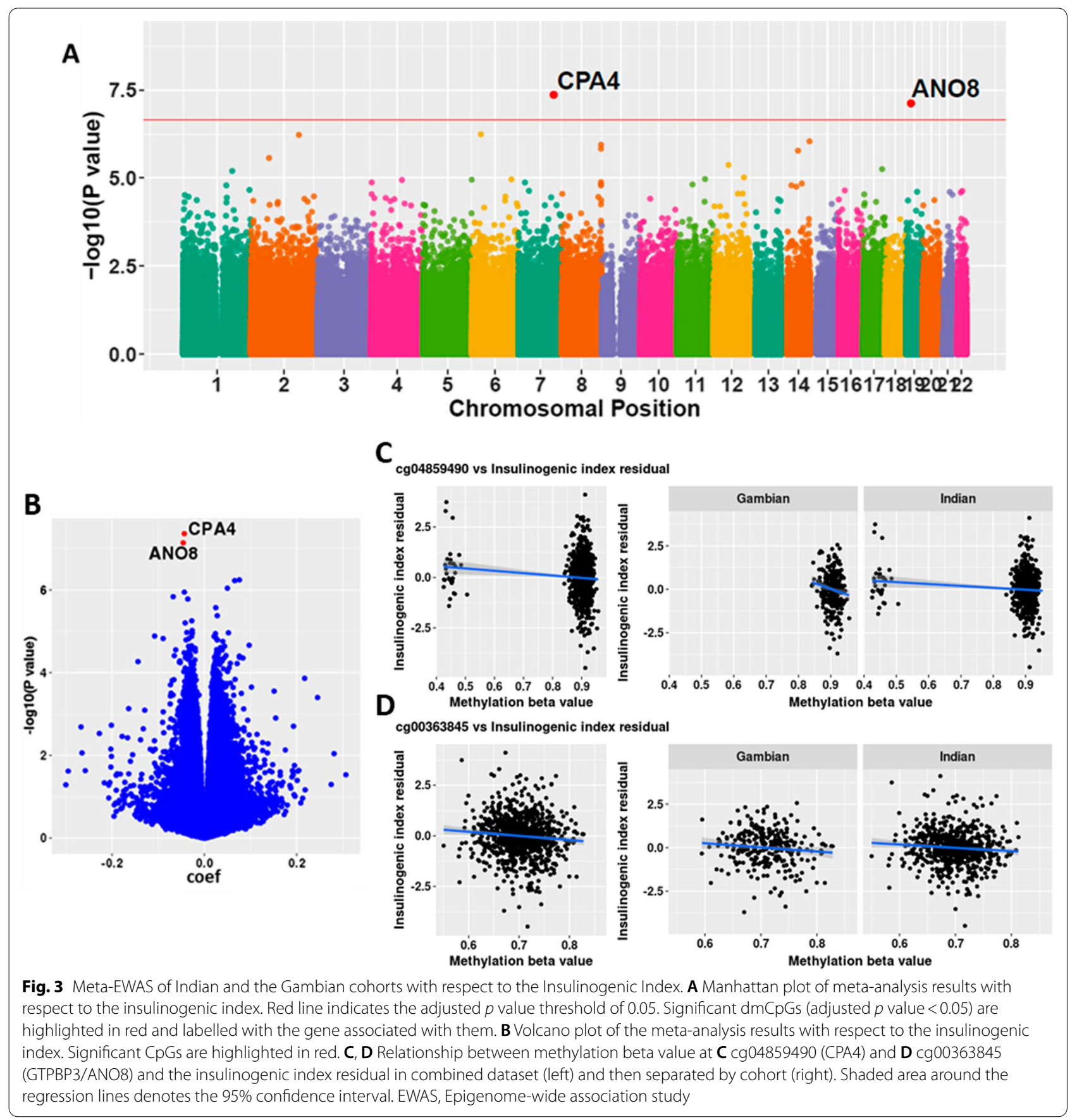

\section{methQTL analysis}

As DNA methylation at specific loci can be influenced by genotype, we used GEM to identify methylation quantitative trait loci (meQTLs) associated with the cardiometabolic dmCpGs identified in the two cohorts. For the Indian cohort, we analysed associations between the 15 CMD identified dmCpGs and 4,312,147 SNPs. No significant methQTLs were identified in cis- or trans- with a Bonferroni threshold of $2.4 \times 10^{-8}$ and $2.8 \times 10^{-9}$, respectively (see Methods for further details). For the Gambian cohort, we analysed associations between the $15 \mathrm{dmCpGs}$ and 4,555,414 SNPs. We identified a total of 79 cis-methQTLs using a Bonferroni threshold of $p=2.4 \times 10^{-8}$ (Additional file 1: Table S4): 44 methQTLs associated with cg00368636, 14 with cg13135286 and 21 with $\operatorname{cg} 13819288$. No trans-methQTLs were identified using a Bonferroni threshold $p=2.8 \times 10^{-9}$. A sensitivity analysis demonstrated that effect sizes reported in the 
main analysis for cg00368636 and cg13819288 were not significantly affected by cis-methQTL effects, while the effect size for the LDL-cholesterol associated cg13135286 was significantly reduced after adjustment for the multiple cis-methQTLs (Additional file 1: Table S5). Additional investigation revealed that seven of the fourteen identified cg13135286-associated methQTLs were directly associated with LDL-cholesterol (Additional file 1: Table S6). We conducted further analyses to investigate the effect of these methQTLs on the reported $\mathrm{dmCpG}$ association (Additional file 4: Figure S3A) and observed that the methQTLs clustered into three linkage disequilibrium (LD) blocks (Additional file 1: Table S7, Additional file 4: Figure S3B, C). A mediation analysis was carried out to determine if the genotype associations with LDL-Cholesterol levels were mediated by methylation at $\operatorname{cg} 13135286$. We found no evidence that cg13135286 mediated the effect of the three tag SNPs (rs75332983, rs614038 and rs6659203) on LDL-Cholesterol levels (Additional file 1: Table S8).

\section{Discussion}

In this study, we report findings from the EMPHASIS study, investigating associations between DNAm in children from two LMICs with concurrent measures of cardiometabolic risk factors. We identified novel methylation changes associated with CMD risk factors including blood pressure, insulin sensitivity and lipids at both single CpG and regional levels in the individual cohorts, and common methylation changes associated with insulin secretion and pulse pressure in a meta-analysis. These findings may provide insights into molecular pathways associated with CMD in two understudied LMIC populations.

EWAS analysis identified associations between DNAm and blood pressure. The meta-analysis identified a $\mathrm{dmCpG}$ within the CIT gene associated with pulse pressure, a measure of arterial stiffness. Furthermore, in the Gambian cohort, we found significant associations between $\mathrm{CpGs}$ associated with the MED22 and EGLN2 genes with SBP; and a DMR, comprising three CpGs, within the EFNA1 gene associated with DBP. While MED22 has not previously been linked to vascular function, EGLN2 is involved in regulating hypoxia tolerance and apoptosis in cardiac and skeletal muscle [23]. EFNA1, an EPH receptor protein-tyrosine kinase, is highly expressed in vascular smooth muscle, triggering EPHA4 signalling and stress fibre assembly [24]. However, no studies have linked the genetic/epigenetic regulation of EFNA1 with blood pressure. In adults, associations between DNAm and blood pressure have been reported in several cohorts $[25,26]$, in which trans-ethnic differences were identified [25]. However, the dmCpGs identified here did not overlap with those reported by Kazmi et al. [25] in Europeans and South Asian men or those reported in a meta-analysis of multiple African American cohorts [26].

The insulinogenic index is a measure of first-phase insulin secretion. In the meta-analysis, there were associations between CpGs within the CPA4 and GTPBP3 genes and insulinogenic index. Carboxypeptidase A4 (CPA4) is an exopeptidase, negatively regulating adipogenesis and downregulated during adipocyte differentiation by FGF1 [27]. CPA4 expression in adipose tissue is inversely correlated with insulin sensitivity, implicating CPA4 in maintaining local and systemic insulin sensitivity [27]. Canonically, DNA methylation across the promoter represses gene expression, while gene body methylation is generally positively associated with expression [28]. This suggests that higher methylation at this $\mathrm{CpG}$ may increase CPA4 expression, resulting in reduced insulin sensitivity driving increased insulin secretion in children with good pancreatic reserve, consistent with the findings reported by He et al. [27]. This is supported by the absence of association with insulin secretion adjusted for insulin sensitivity. GTPBP3 is involved in mitochondrial tRNA modification, with decreased expression associated with reduced oxygen consumption and ATP production [29]. As increased ATP is necessary for the membranedependent increases in cytosolic $\mathrm{Ca}^{2+}$, the main trigger of insulin exocytosis [30], altered epigenetic regulation of GTPBP3 may have downstream effects on insulin secretion. Furthermore, we found associations between DNAm and insulin measures in the individual cohorts. In the Gambian cohort, two dmCpGs were associated with fasting insulin levels, while in the Indian cohort there were associations with HOMA2-S, insulinogenic index adjusted for HOMA2-S and fasting glucose, with no overlap between the cohorts. Many of the dmCpGs and DMRs were located within intergenic regions, so their potential influence on gene expression and on insulin/ glucose homeostasis is currently unclear.

Although there were no associations between DNAm and lipid levels in the meta-analysis, there were associations in the individual cohorts. Six dmCpGs were significantly associated with LDL-Cholesterol levels in the Gambian children, of which cg01469688, located in the promoter of the SCAI gene, was the most significant. $S C A I$ is a transcriptional modulator regulating myocardin, implicated in cardiac hypertrophy [31] and hypertension [32]. There are many possible interpretations of this observation, including that LDL-Cholesterol may influence the epigenetic regulation of this cardiac transcriptional regulator, contributing to the development of CVD. Alternatively, SCAI transcription could influence methylation, with the $\mathrm{dmCpG}$ serving as a biomarker of 
cardiovascular stress associated with LDL-Cholesterol levels. In the Indian cohort, there were no associations of DNAm with triglycerides or LDL-Cholesterol levels. However, cg04988216 within the body of the ROR1 gene was negatively correlated with HDL-Cholesterol levels. ROR1 plays an essential role in skeletal and cardiac development [33]. Moreover, Sánchez-Solana et al. [34] have shown that inhibition of ROR1 modulates ERK1/2 activity in mice, regulating the expression of glucose transporters 1 and 4. Decreased gene body methylation within ROR1 may indicate decreased expression of ROR1, subsequently promoting increased adipogenesis in those with low HDL-Cholesterol levels, affecting susceptibility to later life metabolic disease.

In adults, previous EWAS have identified robust associations between CMD risk markers and DNAm at key genes in lipid metabolism [35]. Moreover, several of these are associated with increased CMD incidence. For example, CpGs within CPT1A were associated with the metabolic syndrome [36] and plasma adiponectin, a biomarker for CMD/CVD risk [37]. A recent Mendelian randomisation study has suggested a causal effect of methylation at $A B C G 1$ on BMI and lipid levels [14]. We did not find associations at these previously reported CpGs in our study, possibly because we measured DNAm in children without overt indications of CMD. Furthermore, the range of physiological and biochemical measures in children is smaller and presumably subject to tighter metabolic homeostasis than in adults, due to the absence of comorbidities. Moreover, the children here are from LMICs where there have been limited epigenetic studies, and differences in genotype and environment may contribute to DNAm differences associated with CMD traits.

The marked differences observed between the results in the two cohorts could reflect potential populationspecific phenotypic differences. Studies have shown that Indians have greater body fat and central obesity compared to black African-Caribbean populations [38, 39]. This is reflected in higher plasma non-esterified fatty acids and triglycerides, hyperinsulinemia and IR during fasting and post-glucose challenge in the Indian population [38]. DNAm differences between the cohorts could influence, or be influenced by, the different distributions of these cardiometabolic markers, and may mark corresponding differences in IR progression.

Differences in DNAm between the populations may also result from differences in diet, environment and/or genotype. The Indian children were living in overcrowded urban slums with high levels of air pollution, which may affect the methylome [40]. In contrast, the Gambian children are from a remote rural area where the food supply is heavily season dependent [22]. However, the dmCpGs identified here were not associated with SoC or maternal pre-conceptional and pregnancy micronutrient intervention [19]. Additionally, we found limited evidence that the $\mathrm{dmCpGs}$ were influenced by measured genetic variation, with only three $\mathrm{dmCpGs}$ in the Gambian analysis having significantly associated methQTLs. We also found no evidence that methylation mediates an effect of genotype at a single CpG in Gambians where both the CpG and methQTLs were associated with LDL-Cholesterol. We note that differences in power due to the varied sample sizes of the two cohorts are unlikely to underlie the contrasting findings since effect sizes and $p$ values are markedly different for $\mathrm{dmCpGs}$ identified in one cohort or the other (Table 2).

There are several strengths to this study. Firstly, we were able to analyse an extensive set of blood-derived markers and phenotype measures on the children, allowing detailed assessment of the relationship between DNAm and CMD markers in childhood. Secondly, investigating associations in children from two LMICs gives an opportunity to assess methylation changes in two understudied populations and for inter-cohort comparison. Although replication in HIC cohorts is possible, environmental and lifestyle differences between LMICs and HICs would be likely to confound the results. Limitations of the study include the lack of suitable cohorts from LMICs with both methylation data and CMD measures in childhood. Furthermore, DNAm was measured in peripheral blood, which has limited relevance to the aetiology of CMD traits. We also have no earlier measures of phenotypes or DNAm in the children, so cannot investigate temporal relationships. However, while we found no evidence of causal relationships, methylation changes presented here, if replicated, might serve as useful biomarkers for identifying individuals at increased risk of CMD.

\section{Conclusions}

We carried out a comprehensive analysis of the relationship between concurrent DNA methylation in peripheral blood and measures of cardiometabolic health in children from two LMICs. We identify both cohort-specific and common associations. With further replication, identified methylation changes during early childhood may serve as biomarkers of future CMD risk and may provide insights into molecular pathways leading to CMD in later life.

\section{Methods}

\section{Study cohorts}

The EMPHASIS study $[18,19]$ comprises two cohorts of children born to mothers who took part in separate randomised controlled trials of nutritional supplementation before and during pregnancy. The original trials were: the Mumbai Maternal Nutrition Project (MMNP) (also 
known as project SARAS-ISRCTN62811278) among women living in slums in the city of Mumbai, India; and the Peri-conceptional Multiple Micronutrient Supplementation Trial (PMMST-ISRCTN13687662) among women living in rural West Kiang, The Gambia. The Mumbai children have been followed up at 5-7 years of age ("SARAS KIDS" study) and data and samples for the first 700 children studied from the per protocol group (children whose mothers started supplementation at least 3 months prior to conception) [41] have been used in the EMPHASIS study. The Gambian children were followed up aged 7-9 years in 2016; all 299 Gambian children retraced from the PMMST group were included in the study. The current investigation is a part of the Stage 2 analysis of the EMPHASIS study [18].

\section{Physiological and biochemical measurements}

Full details of the physiological and biochemical procedures carried out are described in Additional file 5: Methods. Briefly, blood pressure was measured using an Omron HEM 7080 and Omron 705IT in the Gambian and Indian children, respectively. In both cohorts, fasting blood samples were collected. For the oral glucose tolerance test (OGTT), an oral anhydrous glucose load of $1.75 \mathrm{~g} / \mathrm{kg}$ body weight was administered, after which blood samples were collected at 30 and $120 \mathrm{~min}$. In the Gambian cohort, glucose and lipid concentrations were measured using the COBAS INTEGRA ${ }^{\circledR} 400$ plus analyser (Roche Diagnostics, USA). Insulin was measured using the VITROS 350 Analyzer (Ortho Clinical Diagnostics, USA). In the Indian cohort, plasma glucose concentrations were measured using standard enzymatic methods; insulin using ELISA kits (Mercodia, Sweden); and lipids (HDL-/LDL-Cholesterol and triglycerides) using ready-to-use kits (Dialab, Austria).

\section{Derived variables}

Insulin sensitivity (HOMA2-S) was derived from fasting glucose and insulin using the Oxford calculator (https://www.phc.ox.ac.uk/research/technology-outpu ts/ihoma2). Two measures of first-phase insulin secretion were derived: the Insulinogenic index [42] and the Insulinogenic index adjusted for insulin sensitivity, calculated as the residual of insulinogenic index regressed on HOMA2-S. See Additional file 5: Methods for further details.

\section{Processing of outcome variables}

Distributions of physiological variables were checked and log-transformed if necessary. Residuals were generated for all physiological variables by adjusting for the child's age, sex and current height and body mass index (BMI) where such associations were statistically significant and used in the final regression models. Further details are in Additional file 5: Methods.

\section{Epigenome-wide DNA methylation quality control (QC) and pre-processing}

DNA was extracted from peripheral blood samples as previously described [19]. Epigenome-wide DNA methylation profiling was performed for a total of 698 Indian and 293 Gambian samples using the Human MethylationEPIC BeadChip platform (Illumina, USA). Full details of QC and pre-processing are described in Saffari et al. [19]. Briefly, the raw.idat files were processed in $R$ (v3.5.2) using the Bioconductor package meffil [43]. Sex mismatches (5 Indian, 0 Gambian samples) and outlying arrays (7 Indian, 4 Gambian samples) were excluded. Probes with a low detection $\mathrm{p}$ value or bead numbers (1494 and 2635 probes in Indian and Gambian data, respectively), mapping to sex chromosomes and/or cross-reactive (61,523 and 61,225 probes in Indian and Gambian data, respectively) were excluded. After preprocessing and QC, this left 686 samples and 803,120 probes in the Indian cohort, and 289 samples and 802,283 probes in the Gambian cohort.

\section{Epigenome-wide association studies (EWAS) \\ Site-level differential methylation analysis}

For EWAS analysis, robust regression models were run using limma (v3.38.3) [44] with methylation $M$ values as the outcome variable due to their superior distributional properties for linear regression modelling in differential methylation analysis [45]. Models were adjusted for child's sex, age at measurement and the first ten principle components from a methylation principle component analysis (PCA) derived from the 200,000 most variable probes to account for technical covariates and white blood cell composition [19]. The analysis was controlled for multiple testing with the Benjamini-Hochberg adjustment for false discovery rate, with a significance threshold of an FDR $<0.05$. Inflation of $p$ values was assessed (lambda), Quantile-Quantile (Q-Q) plots generated and bacon (v1.10.1) [46] used to control for genomic inflation of test statistics where lambda $>1.2$.

Effect sizes for site-level analysis used methylation beta values to aid interpretation [45]. An additional investigation of interactions between methylation and sex was carried out for significant differentially methylated CpGs (dmCpGs) only.

\section{Regional-level differential methylation analysis}

The methylation status of adjacent CpG sites can be highly correlated, often with functional relevance and analysis of regional changes in methylation can provide increased statistical power. DMRcate (v1.18.0) [47] was 
used for the identification of differentially methylated regions (DMRs) with respect to the different measures of cardiometabolic health. DMRcate uses Stouffer's method for combining $\mathrm{p}$ values for CpGs, with a Stouffer $<0.05$ threshold being used as statistically significant [19].

\section{Sensitivity analyses}

To identify sex dependent methylation effects, we investigated potential sex-interaction effects (outcome $\mathrm{x}$ child sex) on the significant $\mathrm{dmCpGs}$ identified in the main analysis using the following regression model:

$$
\begin{aligned}
& \text { CpG_beta } \sim \text { Physiological Outcome }+ \text { child sex } \\
& \quad+\text { outcome } * \text { child sex }+ \text { child age } \\
& +10 \text { principle components }
\end{aligned}
$$

Furthermore, it has previously been reported that season of conception in The Gambia influences infant's DNA methylation and early growth. Therefore, the effect of season of conception (SoC) on the outcome-associated loci was tested by inclusion as a covariate in the main regression model and secondly, by inclusion of an interaction between DNA methylation and $\mathrm{SoC}$ as below:

$$
\begin{aligned}
& \text { Outcome } \sim \text { CpG_beta }+ \text { SoC }+ \text { CpG_beta } * \text { SoC } \\
& + \text { child sex }+ \text { child age }+10 \text { principle components }
\end{aligned}
$$

A Bonferroni-adjusted significant threshold: $p<2.8 \times 10^{-3}(0.05 / 18)$ was used to adjust for multiple testing of the $18 \mathrm{dmCpGs}$ investigated for sex interactions.

\section{Meta-analysis}

To examine common associations across cohorts, effect size estimates from individual EWAS were meta-analysed using METAL [48] with an inverse variance weighting. Correction for inflation of both input and meta-analysis output statistics was performed using double genomic control (GC). We explored heterogeneity between the two cohorts using the $I^{2}$ statistic $[49,50]$.

\section{Genotyping}

SNP genotypes for 293 Gambian and 698 Indian samples were generated using the Infinium Global Screening Array-24 v1.0 BeadChip array (Illumina, USA) and imputed against a 1000 Genomes Phase 3 reference panel using IMPUTE2 (v2.3.2). Full details of QC and pre-processing can be found in Saffari et al. [19] and in Additional file 5: Methods. The final imputed data sets comprised 284 samples with 4,555,414 SNPs in the Gambian cohort, and 686 samples with 4,312,147 SNPs in the Indian cohort.

\section{Methylation quantitative trait loci (methQTL) analysis} methQTL analysis was carried out by the GEM package (v1.10.0), using an additive model [17]. The analysis was restricted to the significant $\mathrm{dmCpGs}$ identified in single cohort and/or meta-analyses. Separate analyses in cis ( $\mathrm{SNP} \pm 2 \mathrm{Mb}$ from the $\mathrm{CpG}$ ) and trans (all other SNPs) were conducted to maximise power. Significant cis-methQTLs were those with a Bonferroni-adjusted $p$ value $<2.4 \times 10^{-8}$, while trans-methQTLs were those with a Bonferroni-adjusted $p<2.8 \times 10^{-9}$. Full details are described in Additional file 5: Methods.

In order to minimise non-genetic variation in the DNA methylation data, we regressed out the effect of child sex, plus the first 10 principal components (PCs) from an unsupervised PCA of the methylation data, prior to performing the methQTL analysis. The resulting methylation residuals were then rank transformed and centred to have mean 0 and variance 1 [51]. The methQTL analysis was carried out using the GEM package (v1.10.0) from Bioconductor which uses an additive (allelic dose) model to test for SNP-methylation associations [17]. This analysis was restricted to all $18 \mathrm{CpGs}$ identified in single cohort and/or meta-analyses.

Separate analyses in cis ( $\mathrm{SNP} \pm 2 \mathrm{Mb}$ from the $\mathrm{CpG})$ and trans (all other SNPs) were conducted to maximise power. Significant $c i s$-methQTLs were those with a Bonferroni-adjusted $p$ value $<2.4 \times 10^{-8}$, while transmethQTLs were those with a Bonferroni-adjusted $p<2.8 \times 10^{-9}$.

i. Identification of cis-methQTL

Only SNPs within $+/-2 \mathrm{MB}$ of a $\mathrm{CpG}$ of interest were considered for this analysis. Significant cis-methQTL are those with an association $\mathrm{p}$ value passing a Bonferroni-adjusted significance threshold of $p=0.05 / \mathrm{n}$ SNPs/n_CpGs, where $n \_S N P s$ is the number of cis-SNPs and $n_{-}$CpGs is the total number of CpGs tested ( $n=18$ CpGs).

ii. Identification of trans-methQTL

trans-methQTL are methQTL with an association $\mathrm{p}$ value passing a genome-wide Bonferroni-adjusted threshold of $p=5 \times 10^{-8} / \mathrm{n}$ _CpGs that do not fall within the set of cis-methQTL identified in i) above.

For CpGs with significant methQTLs, we conducted a sensitivity analysis to see if methQTL effects significantly changed effect sizes for the CpG-outcome associations reported in the main EWAS analysis. We did this by repeating the main analysis with the methQTLSNP as an additional covariate and comparing original effect size estimates with $95 \%$ confidence intervals for the effect size in the adjusted model.

We also investigated associations between identified methQTLs and the traits associated with the methQTLassociated $\mathrm{CpG}$ identified in the main EWAS, using 
an additive (allelic dose) model with methylation values transformed as above. For CpGs with significant methQTL-trait associations, we tested the potential for this association to be mediated by methylation changes at the associated $\mathrm{CpG}$ using the mediate() function from the mediation package [52]. Confidence intervals for direct and indirect (mediated) effects were calculated using a nonparametric bootstrap with 1000 simulations.

\begin{abstract}
Abbreviations
CMD: Cardiometabolic disease; CVD: Cardiovascular disease; LMIC: Low- and middle-income countries; HIC: High-income countries; DNAm: DNA methylation; dmCpG: Differentially methylated CpG; DMR: Differentially methylated region; SBP: Systolic blood pressure; DBP: Diastolic blood pressure; PP: Pulse pressure; HDL-C: High-density lipoprotein cholesterol; LDL-C: Low-density lipoprotein cholesterol; methQTL: Methylation quantitative trait loci; FDR: False discovery rate; EWAS: Epigenome-wide association study.
\end{abstract}

\section{Supplementary Information}

The online version contains supplementary material available at https://doi. org/10.1186/s13148-021-01213-3.

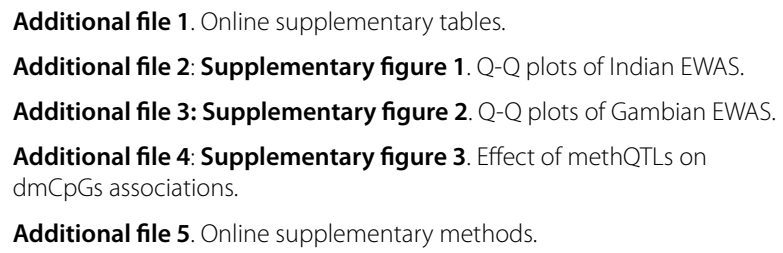

Additional file 5. Online supplementary methods.

\section{Acknowledgements}

We would like to thank all the EMPHASIS study participants in Mumbai, India and West Kiang, The Gambia for their time and commitment. We also thank members of the laboratory and field teams working in both countries. Finally, we are grateful to our partners and advisors Caroline Relton (steering committee chair), Partha P Majumder and Frank Dudbridge (steering committee members). Other members of the EMPHASIS Study Group: Sarah Kehoe (MRC Lifecourse Epidemiology Unit, University of Southampton, Southampton, UK), Kalyanaraman Kumaran (MRC Lifecourse Epidemiology Unit, University of Southampton, Southampton, UK; CSI Holdsworth Memorial Hospital, Mysore, India), Ramesh D. Potdar (Centre for the Study of Social Change, Mumbai, India), Sara Sajjadi (Genomic Research on Complex Diseases (GRC Group), CSIR - Centre for Cellular and Molecular Biology, Hyderabad, India), Suraj Nongmaithem (Genomic Research on Complex Diseases (GRC Group), CSIR_Centre for Cellular and Molecular Biology, Hyderabad, India), Harsha Chopra (Centre for the Study of Social Change, Mumbai, India), Harshad Sane (Centre for the Study of Social Change, Mumbai, India), Meera Gandhi (Centre for the Study of Social Change, Mumbai, India), Stephen Owens (Institute of Health and Society, Newcastle University, Newcastle, UK), Landing Jarjou (MRC Unit The Gambia at the London School of Hygiene and Tropical Medicine, UK), Ann Prentice (MRC Unit The Gambia at the London School of Hygiene and Tropical Medicine, UK).

\section{Authors' contributions}

C.H.D.F., G.R.C., M.J.S. and A.M.P. conceived and designed the study; P.I., S.S., A.S.T. and M.B. performed the methylation and laboratory assays; S.A.S. and M.B. conducted sample and data collection; A.S., S.S., P.I., M.A., A.S.T., P.J., M.J.S., G.R.C., C.H.D.F. and K.A.L. devised the analysis strategy; A.S., E.A., P.I., S.S., A.S.T., C.d.G., M.J.S. and M.A. performed statistical and bioinformatics analyses; E.A., P.I., S.S., M.J.S., G.R.C., C.H.D.F. and K.A.L. drafted the manuscript and all authors critically revised and approved the manuscript. All authors read and approved the final manuscript.

\section{Funding}

MMNP was supported by the Wellcome Trust, Parthenon Trust, ICICI Bank Ltd., Mumbai, the UK Medical Re-search Council (MRC) and the UK Department for International Development (DFID) under the MRC/DFID Concordat. The children's follow-up (SARAS KIDS) was funded by MRC research grant MR/ M005186/1. PMMST was supported by MRC (U1232661351, U105960371 and MC-A760-5QX00) and DFID under the MRC/DFID Concordat, and other members of the Gambian team were supported by MRC grants U105960371, U123261351 and MR/M01424X/1. The EMPHASIS study was jointly funded by MRC, DFID and the Department of Biotechnology (DBT), Ministry of Science and Technology, India, under the Newton Fund initiative (MRC Grant No.: MR/ N006208/1 and DBT Grant No.: BT/IN/DBT-MRC/DFID/24/GRC/2015-16).

\section{Availability of data and materials}

EPIC data have been placed on the server of the CSIR-Centre for Cellular and Molecular Biology in Hyderabad and can be accessed with permission from the authors. Bespoke code written for the analysis is available at https:// github.com/asaffa/EMPHASIS.

\section{Declarations}

\section{Ethics approval and consent to participate}

MMNP (ISRCTN62811278) was approved by the ethics committees of BYL Nair and TN Medical College, Grant Medical College, and Sir JJ Group of Hospitals, Mumbai. PMMST (ISRCTN13687662) was approved by the joint Gambia Government/Medical Research Council (MRC) Unit The Gambia's Ethics Committee. Ethics approval for the follow-up of the children in Mumbai ("SARAS KIDS") was obtained from the Intersystem Biomedica Ethics Committee, Mumbai on 31 May 2013 (serial no. ISBEC/NR-54/KM/JVJ/2013). Ethics approval for the EMPHASIS study in The Gambia was obtained from the joint Gambia Government/MRC Unit The Gambia's Ethics Committee on 19 October 2015 (serial no. SCC 1441). The EMPHASIS study is registered as ISRCTN14266771. Signed informed consent was obtained from parents and verbal assent from the children.

\section{Consent for publication}

Not applicable.

\section{Competing interests}

The authors declare that they have no competing interests.

\section{Author details}

${ }^{1}$ School of Medicine, University of Southampton, Southampton, UK. ${ }^{2}$ Genomic Research On Complex Diseases (GRC Group), CSIR-Centre for Cellular and Molecular Biology, Hyderabad, India. ${ }^{3}$ MRC Lifecourse Epidemiology Unit, University of Southampton, Southampton, UK. ${ }^{4}$ School of Basic and Applied Sciences, Dayananda Sagar University, Bangalore, India. ${ }^{5}$ MRC Unit The Gambia at the London, School of Hygiene and Tropical Medicine, Fajara, The Gambia. ${ }^{6} \mathrm{MRC}$ Unit The Gambia at the London, School of Hygiene and Tropical Medicine, London, UK. ${ }^{7}$ Centre for the Study of Social Change, Mumbai, India. ${ }^{8}$ Biological Sciences, University of Southampton, Southampton, UK.

Received: 28 September 2021 Accepted: 8 December 2021

Published online: 09 January 2022

\section{References}

1. Dunachie S, Chamnan P. The double burden of diabetes and global infection in low and middle-income countries. Trans R Soc Trop Med Hyg. 2019;113(2):56-64.

2. Yeates K, Lohfeld L, Sleeth J, Morales F, Rajkotia Y, Ogedegbe O. A global perspective on cardiovascular disease in vulnerable populations. Can J Cardiol. 2015;31(9):1081-93.

3. Collaboration NCDRF. Worldwide trends in diabetes since 1980: a pooled analysis of 751 population-based studies with 4.4 million participants. Lancet. 2016;387(10027):1513-30. 
4. Patel A, Bharani A, Sharma M, Bhagwat A, Ganguli N, Chouhan DS. Prevalence of hypertension and prehypertension in schoolchildren from Central India. Ann Pediatr Cardiol. 2019;12(2):90-6.

5. Madhivanan SHE, Kumarasamy K. A study of blood pressure in school children between the age of 6-12 years in Chennai, India: a cross sectional study. Int J Contemp Pediatrics. 2017;4(5):2205-12.

6. Group NCDRFC-AW. Trends in obesity and diabetes across Africa from 1980 to 2014: an analysis of pooled population-based studies. Int J Epidemiol. 2017:46(5):1421-32.

7. Luhar S, Mallinson PAC, Clarke L, Kinra S. Trends in the socioeconomic patterning of overweight/obesity in India: a repeated cross-sectional study using nationally representative data. BMJ Open. 2018;8(10):e023935.

8. Godfrey KM, Inskip HM, Hanson MA. The long-term effects of prenatal development on growth and metabolism. Semin Reprod Med. 2011;29(3):257-65.

9. Fleming TP, Watkins AJ, Velazquez MA, Mathers JC, Prentice AM, Stephenson J, et al. Origins of lifetime health around the time of conception: causes and consequences. Lancet. 2018:391(10132):1842-52.

10. Gluckman PD, Hanson MA, Beedle AS, Spencer HG. Predictive adaptive responses in perspective. Trends EndocrinolMetab. 2008:19(4):109-10.

11. Lillycrop KA, Phillips ES, Jackson AA, Hanson MA, Burdge GC. Dietary protein restriction of pregnant rats induces and folic acid supplementation prevents epigenetic modification of hepatic gene expression in the offspring. J Nutr. 2005;135(6):1382-6.

12. Geraghty AA, Lindsay KL, Alberdi G, McAuliffe FM, Gibney ER. Nutrition during pregnancy impacts offspring's epigenetic status-evidence from human and animal studies. Nutr Metab Insights. 2015;8(Suppl 1):41-7.

13. James P, Sajjadi S, Tomar AS, Saffari A, Fall CHD, Prentice AM, et al. Candidate genes linking maternal nutrient exposure to offspring health via DNA methylation: a review of existing evidence in humans with specific focus on one-carbon metabolism. Int J Epidemiol. 2018:47(6):1910-37.

14. Mendelson MM, Marioni RE, Joehanes R, Liu C, Hedman AK, Aslibekyan $\mathrm{S}$, et al. Association of body mass index with dna methylation and gene expression in blood cells and relations to cardiometabolic disease: a Mendelian randomization approach. PLoS Med. 2017;14(1):e1002215.

15. Al Muftah WA, Al-Shafai M, Zaghlool SB, Visconti A, Tsai PC, Kumar P, et al. Epigenetic associations of type 2 diabetes and BMI in an Arab population. Clin Epigenetics. 2016;8:13.

16. Kho M, Zhao W, Ratliff SM, Ammous F, Mosley TH, Shang L, et al. Epigenetic loci for blood pressure are associated with hypertensive target organ damage in older African Americans from the genetic epidemiology network of Arteriopathy (GENOA) study. BMC Med Genomics. 2020;13(1):131.

17. Pan H, Holbrook JD, Karnani N, Kwoh CK. Gene, Environment and Methylation (GEM): a tool suite to efficiently navigate large scale epigenome wide association studies and integrate genotype and interaction between genotype and environment. BMC Bioinform. 2016;17:299.

18. Chandak GR, Silver MJ, Saffari A, Lillycrop KA, Shrestha S, Sahariah SA, et al. Protocol for the EMPHASIS study; epigenetic mechanisms linking maternal pre-conceptional nutrition and children's health in India and Sub-Saharan Africa. BMC Nutr. 2017;3.

19. Saffari A, Shrestha S, Issarapu P, Sajjadi S, Betts M, Sahariah SA, et al. Effect of maternal preconceptional and pregnancy micronutrient interventions on children's DNA methylation: findings from the EMPHASIS study. Am J Clin Nutr. 2020;112(4):1099-113.

20. Godfrey KM, Costello PM, Lillycrop KA. Development, epigenetics and metabolic programming. Nestle Nutr Inst Workshop Ser. 2016;85:71-80.

21. Joubert BR, Felix JF, Yousefi P, Bakulski KM, Just AC, Breton C, et al. DNA methylation in newborns and maternal smoking in pregnancy: genomewide consortium meta-analysis. Am J Hum Genet. 2016:98(4):680-96.

22. Dominguez-Salas P, Moore SE, Baker MS, Bergen AW, Cox SE, Dyer RA, et al. Maternal nutrition at conception modulates DNA methylation of human metastable epialleles. Nat Commun. 2014;5:3746.

23. Epstein AC, Gleadle JM, McNeill LA, Hewitson KS, O'Rourke J, Mole DR, et al. C. elegans EGL-9 and mammalian homologs define a family of dioxygenases that regulate HIF by prolyl hydroxylation. Cell. 2001;107(1):43-54.

24. Wu Z, Luo H, Thorin E, Tremblay J, Peng J, Lavoie JL, et al. Possible role of Efnb1 protein, a ligand of Eph receptor tyrosine kinases, in modulating blood pressure. J Biol Chem. 2012;287(19):15557-69.
25. Kazmi N, Elliott HR, Burrows K, Tillin T, Hughes AD, Chaturvedi N, et al. Associations between high blood pressure and DNA methylation. PLoS One. 2020;15(1):e0227728.

26. Richard MA, Huan T, Ligthart S, Gondalia R, Jhun MA, Brody JA, et al. DNA methylation analysis identifies loci for blood pressure regulation. Am J Hum Genet. 2017;101(6):888-902.

27. He J, Chen DL, Samocha-Bonet D, Gillinder KR, Barclay JL, Magor GW, et al. Fibroblast growth factor-1 (FGF-1) promotes adipogenesis by downregulation of carboxypeptidase A4 (CPA4) - a negative regulator of adipogenesis implicated in the modulation of local and systemic insulin sensitivity. Growth Factors. 2016;34(5-6):210-6.

28. Ball MP, Li JB, Gao Y, Lee JH, LeProust EM, Park IH, et al. Targeted and genome-scale strategies reveal gene-body methylation signatures in human cells. Nat Biotechnol. 2009;27(4):361-8.

29. Villarroya M, Prado S, Esteve JM, Soriano MA, Aguado C, Perez-Martinez D, et al. Characterization of human GTPBP3, a GTP-binding protein involved in mitochondrial tRNA modification. Mol Cell Biol. 2008;28(24):7514-31.

30. Maechler P, Wollheim CB. Mitochondrial signals in glucose-stimulated insulin secretion in the beta cell. J Physiol. 2000;529(Pt 1):49-56.

31. Parmacek MS. Myocardin-related transcription factors: critical coactivators regulating cardiovascular development and adaptation. Circ Res. 2007;100(5):633-44.

32. Kontaraki JE, Marketou ME, Zacharis EA, Parthenakis FI, Vardas PE. Early cardiac gene transcript levels in peripheral blood mononuclear cells in patients with untreated essential hypertension. J Hypertens. 2011;29(4):791-7.

33. Nomi M, Oishi I, Kani S, Suzuki H, Matsuda T, Yoda A, et al. Loss of mRor1 enhances the heart and skeletal abnormalities in mRor2-deficient mice: redundant and pleiotropic functions of mRor 1 and mRor2 receptor tyrosine kinases. Mol Cell Biol. 2001;21(24):8329-35.

34. Sanchez-Solana B, Laborda J, Baladron V. Mouse resistin modulates adipogenesis and glucose uptake in 3T3-L1 preadipocytes through the ROR1 receptor. Mol Endocrinol. 2012;26(1):110-27.

35. Pfeiffer L, Wahl S, Pilling LC, Reischl E, Sandling JK, Kunze S, et al. DNA methylation of lipid-related genes affects blood lipid levels. Circ Cardiovasc Genet. 2015;8(2):334-42.

36. Das M, Sha J, Hidalgo B, Aslibekyan S, Do AN, Zhi D, et al. Association of DNA Methylation at CPT1A Locus with Metabolic Syndrome in the Genetics of Lipid Lowering Drugs and Diet Network (GOLDN) Study. PLoS One. 2016;11(1):e0145789.

37. Aslibekyan S, Do AN, Xu H, Li S, Irvin MR, Zhi D, et al. CPT1A methylation is associated with plasma adiponectin. Nutr Metab Cardiovasc Dis. 2017;27(3):225-33.

38. McKeigue PM, Shah B, Marmot MG. Relation of central obesity and insulin resistance with high diabetes prevalence and cardiovascular risk in South Asians. Lancet. 1991;337(8738):382-6.

39. Shaw NJ, Crabtree NJ, Kibirige MS, Fordham JN. Ethnic and gender differences in body fat in British schoolchildren as measured by DXA. Arch Dis Child. 2007:92(10):872-5.

40. Rider CF, Carlsten C. Air pollution and DNA methylation: effects of exposure in humans. Clin Epigenetics. 2019:11(1):131.

41. Potdar RD, Sahariah SA, Gandhi M, Kehoe SH, Brown N, Sane H, et al. Improving women's diet quality preconceptionally and during gestation: effects on birth weight and prevalence of low birth weight-a randomized controlled efficacy trial in India (Mumbai Maternal Nutrition Project). Am J Clin Nutr. 2014;100(5):1257-68.

42. Phillips DI, Clark PM, Hales CN, Osmond C. Understanding oral glucose tolerance: comparison of glucose or insulin measurements during the oral glucose tolerance test with specific measurements of insulin resistance and insulin secretion. Diabet Med. 1994:11(3):286-92.

43. Min JL, Hemani G, Davey Smith G, Relton C, Suderman M. Meffil: efficient normalization and analysis of very large DNA methylation datasets. Bioinformatics. 2018;34(23):3983-9.

44. Ritchie ME, Phipson B, Wu D, Hu Y, Law CW, Shi W, et al. limma powers differential expression analyses for RNA-sequencing and microarray studies. Nucleic Acids Res. 2015;43(7):e47.

45. Du P, Zhang X, Huang CC, Jafari N, Kibbe WA, Hou L, et al. Comparison of Beta-value and $\mathrm{M}$-value methods for quantifying methylation levels by microarray analysis. BMC Bioinform. 2010;11:587. 
46. van Iterson M, van Zwet EW, Consortium B, Heijmans BT. Controlling bias and inflation in epigenome- and transcriptome-wide association studies using the empirical null distribution. Genome Biol. 2017;18(1):19.

47. Peters TJ, Buckley MJ, Statham AL, Pidsley R, Samaras K, RVL, et al. De novo identification of differentially methylated regions in the human genome. Epigenetics Chromatin. 2015;8:6.

48. Willer CJ, Li Y, Abecasis GR. METAL: fast and efficient meta-analysis of genomewide association scans. Bioinformatics. 2010;26(17):2190-1.

49. Higgins JP, Thompson SG. Quantifying heterogeneity in a meta-analysis. Stat Med. 2002;21(11):1539-58.

50. Cumpston M, LiT, Page MJ, Chandler J, Welch VA, Higgins JP, et al. Updated guidance for trusted systematic reviews: a new edition of the Cochrane Handbook for Systematic Reviews of Interventions. Cochrane Database Syst Rev. 2019;10:ED000142.

51. Mocali S, Chiellini C, Fabiani A, Decuzzi S, de Pascale D, Parrilli E, et al. Ecology of cold environments: new insights of bacterial metabolic adaptation through an integrated genomic-phenomic approach. Sci Rep. 2017;7(1):839.

52. Tingley DYT, Hirose K, Keele L, Imai K. Mediation: R package for causal mediation analysis. J Stat Softw. 2014;59(5):1-38.

\section{Publisher's Note}

Springer Nature remains neutral with regard to jurisdictional claims in published maps and institutional affiliations.

- fast, convenient online submission

- thorough peer review by experienced researchers in your field

- rapid publication on acceptance

- support for research data, including large and complex data types

- gold Open Access which fosters wider collaboration and increased citations

- maximum visibility for your research: over $100 \mathrm{M}$ website views per year

At BMC, research is always in progress.

Learn more biomedcentral.com/submissions 